\title{
Validez y confiabilidad de sonda electroacústica para el diagnóstico de displasia de cadera en neonatos
}

Hernández-Campos Jorge Armando*, Padilla-Raygoza Nicolás**



\section{Introducción}

La displasia de cadera (DC) agrupa un espectro de anormalidades que incluyen displasia del acetábulo y mala posición de la cabeza del fémur, alterando así la estructura y mecánica funcional de dicha articulación; en los casos severos la cabeza femoral no está en contacto con el acetábulo denominándose dislocación o luxación de cadera. ${ }^{1}$ Su presentación incluye displasia de cadera (incongruencia estructural con desarrollo insuficiente del acetábulo), cadera luxable (la cabeza del fémur yace sobre el acetábulo pero puede dislocarse de manera provocada), subluxación (la cabeza femoral está * Estudiante del noveno semestre de la carrera de Medicina del Centro de Ciencias de la Salud de la Universidad Autónoma de Aguascalientes, México
** Profesor del Departamento de Enfermería y Obstetricia de la División de Ciencias de la Salud e Ingenierías del Campus Celaya-Salvatierra, Universi-
dad de Guanajuato, México

Fecha de recibido; 3 de abril 2017

Fecha de aceptación: 30 de junio 2017

Correspondencia: Dr Nicolás Padilla-Raygoza. Departamento de Enfermería y Obstetricia de la División de Ciencias de la Salud e Ingenierías del Campus Celaya-Salvatierra, Universidad de Guanajuato, Mutualismo \#303, Colonia Enrique Colunga, CP 38060, Celaya, Guanajuato, México. Teléfono *52 (473) 7320006 correo electrónico www.celayasalvatierra.ugto.mx 
parcialmente fuera de articulación con el acetábulo) o luxación de cadera.

En México, 1\% de los neonatos tiene DC y la evolución a luxación sólo se presenta en 1:7000 nacidos vivos. ${ }^{2,3}$ En la guía de práctica clínica de la "American Academy of Orthopeadic Surgeons 2014 " se reporta frecuencia de displasia en neonatos del $20 \%$, con predominio del sexo femenino (67.7\%), asociación a antecedente de presentación pélvica en $15.2 \%$ y oligohidramnios en $11.6 \%$; la cadera izquierda fue más afectada $(44.4 \%){ }^{4}$

La patogénesis es multifactorial e incluye laxitud anormal de los ligamentos y la capsula articular, limitación del espacio y restricción del movimiento (oligohidramnios), primera gesta, flexión extrema de la cadera con extensión de rodilla, contractura del músculo psoas iliaco. ${ }^{5}$

Actualmente no existen lineamentos universales para el abordaje de este problema de salud, de ahí la importancia de definir los criterios que sirvan para la detección, el diagnóstico y el tratamiento del DC en los tres niveles de atención médica. ${ }^{6,7}$ En la guía de práctica clínica Diagnóstico oportuno de displasia del desarrollo de cadera de CENETEC se clasifica la sospecha de DC en riesgo bajo (varones sin factores de riesgo o con AHF positivos), intermedio (mujeres sin factores de riesgo o varones con presentación pélvica) 0 alto (mujeres con AHF positivos o con presentación pélvica). ${ }^{8}$

En neonatos, el diagnóstico depende de demostrar la inestabilidad de la unión articular (maniobras de Ortolani, Barlow, Galeazzi, Pistón, radiología, pliegues cutáneos, transmisión del sonido y ultrasonografía). El ultrasonido es ampliamente utilizado y se considera el estándar de oro para el diagnóstico (Técnica de Graf) pero suele sobrediagnosticar la enfermedad. ${ }^{9}$ Los lactantes menores desarrollan contracturas limitando la abducción a menos de $90^{\circ}$; las rodillas están a diferentes alturas y las radiografías son positivas. El lactante mayor con DC presenta claudicación indolora y sacudida del lado afectado. En bipedestación la pelvis se inclina hacia el lado opuesto (signo de Trendelenburg para luxación unilateral). En luxación bilateral la pérdida de la abducción es simétrica, hay marcha contoneante, perineo ampliado y contractura de flexión por desplazamiento posterior de la cabeza del fémur. ${ }^{1,9}$

El estándar de oro es el ultrasonido de caderas con técnica de Graf (USGGraf). El tratamiento aún es posible hasta los dos años de vida pero no está cerca de ser tan bueno como en niños que reciben el tratamiento más tempranamente por lo que la piedra angular de buen pronóstico es el diagnóstico precoz.

En aras de fortalecer la detección oportuna y temprana de DC buscamos la validación de un instrumento diagnóstico que permita evaluar de una manera objetiva y precisa la inestabilidad de la articulación en cualquier grado. La sonda electroacústica consiste en un diapasón electroacústico capaz de producir señales sinodales de 


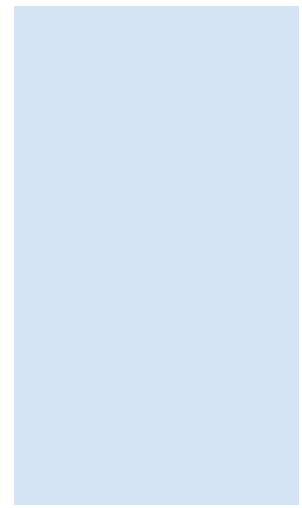

$1 \mathrm{~Hz}-400 \mathrm{kHz}$, transmitidas a la extremidad inferior desde la rótula hasta la sínfisis del pubis; ahí las ondas son captadas por un receptor para su traducción, amplificación y presentación en la pantalla como decibeles o gráficos (transmisión comparada del sonido en extensión/flexión [TCSE/F] con sonda electroacústica).

El objetivo del estudio fue identificar la validez y confiabilidad de la TCSE/F con sonda electroacústica para el diagnóstico de DC en neonatos; para ello calculamos sensibilidad, especificidad, valores predictivos positivos y negativos de nuestra herramienta con USGGraf dinámica (sensibilidad: 95\%, especificidad: 90\%, VPN: $98 \%)^{10,11}$

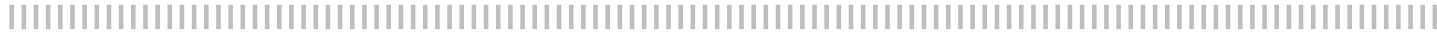

\section{Material y métodos}

El protocolo fue aprobado por el comité de bioética de la división de Ciencias de la Salud e Ingenierías del campus Celaya Salvatierra de la Universidad de Guanajuato. Es un estudio diagnóstico basado en la continuidad. Se realizó en Celaya, Guanajuato, entre junio y julio de 2015.

En el estudio se incluyeron a sujetos que cumplieran con los siguientes criterios: hombres y mujeres de 4 a 28 días de edad, nacidos en el Hospital General de Celaya, Guanajuato, entre el periodo agosto-diciembre de 2014, cuyos padres aceptaron la participación de su hijo (a) en el estudio bajo consentimiento informado por escrito; se excluyeron a neonatos con luxación de cadera embriológica, a los hijos de padres que no quisieron participar y quienes no cumplieran los criterios de inclusión antes mencionados. A los participantes se les midió el peso y la estatura; se interrogó a los padres acerca de la fecha de nacimiento, sexo, peso y estatura al nacer, además de la zona de residencia, y se les dio a firmar consentimiento informado para la participación de su hijo (a) en el estudio. Posteriormente se les aplicó la prueba TCSE/F con sonda electroacústica y después un ultrasonografista experimentado realizó USGGraf (cegado a los resultados de la prueba del sonido).

Para la medición de TCSE/F con sonda electroacústica colocamos al neonato en posición decúbito dorsal sobre la mesa de exploración con las extremidades inferiores en extensión; el diapasón se sitúa sobre rótula derecha y el receptor en la sínfisis del pubis; se genera la onda sonora durante 5 segundos y ésta es captada por el receptor, traducida y presentada en la pantalla. Posteriormente, se flexiona la cadera a $90^{\circ}$ y se realiza nuevamente la medición; si el sonido aumenta a la flexión es indicativo de una alteración en la articulación de la cadera. Realizamos lo mismo para la cadera izquierda colocando el diapasón sobre la rótula izquierda. El procedimiento se aplicó en dos ocasiones por el mismo investigador y una tercera vez por un investigador diferente, esto para conocer la confiabilidad intra e inter-observador.

Cuando las extremidades pélvicas están en extensión y abducción, la transmisión del sonido desde el fémur hasta la sínfisis del pubis es igual en ambos lados y no se modifica a la flexión. Frente a una cadera inestable, subluxada o luxada la transmisión del sonido es menor pero incrementa al flexionar la cadera a $90^{\circ}$ (la relación entre cabeza del fémur y acetábulo aumenta). ${ }^{12}$

La sensibilidad, especificidad y valores predictivos negativo y positivo de la sonda se calcularon utilizando como estándar de oro la ultrasonografía de caderas con 
técnica de Graf (USGGraf con USG portátil Honda MS2000 de traductor plano); el criterio diagnóstico fue el siguiente: Graf I $\left(a>60^{\circ}\right.$ y $\left.b<55^{\circ}\right)$ cadera sana, Graf II $\left(a=44-59^{\circ}\right.$ y $\left.b=55-77^{\circ}\right)$ inmadurez fisiológica, Graf III y IV $\left(a<43^{\circ}\right.$ y b $\left.>77^{\circ}\right)$ subluxación o luxación de cadera. ${ }^{13}$

Se calculó el tamaño de muestra, esperando una sensibilidad del $85 \%$ con una prevalencia de displasia de cadera del 10\%; el tamaño mínimo es de 11 neonatos con $95 \%$ de precisión y $90 \%$ de poder (Epidat3.1, 2005, Xunta de Galicia y OPS). El tamaño de la muestra de nuestro estudio incluye a 100 neonatos de Celaya, Guanajuato. Se utilizó estadística descriptiva para las variables del estudio. La validez se calculó con sensibilidad especificidad y valores predictivos de la prueba de transmisión comparada de sonido en extensión/ flexión con la sonda electroacústica, comparándolas con el estándar de oro que es el USGGraf. Para la confiabilidad se calculó kappa intra-observador (medición 1 vs 2), y kappa inter-observador (medición 1 vs 3). El cálculo estadístico se realizó en STATA 13.0® (Stata Corp., College Station, TX, USA).

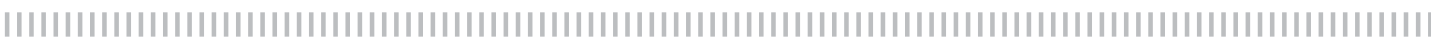

\section{Resultados}

La muestra estuvo integrada por 100 neonatos con una taza de participación de $50 \%$. De éstos, $36 \%$ fue del sexo masculino y $64 \%$ del femenino; $72 \%$ reside en zona urbana, $19 \%$ en suburbana y $9 \%$ en rural. Sólo $16 \%$ de los neonatos reporta antecedentes heredofamiliares de displasia del desarrollo de cadera, siendo los padres los más frecuentemente afectados y $9 \%$ otros familiares. Las características sociodemográficas se muestran en la tabla 1.

\section{Tabla |}

Características sociodemográficas categóricas de los neonatos, Celaya 2014.

\begin{tabular}{|llll|}
\hline Variable & & $\mathbf{n}$ & \% \\
\hline Sexo & Masculino & 36 & 36.00 \\
\hline & Femenino & 64 & 64.00 \\
\hline Residencia & Urbano & 72 & 72.00 \\
\hline & Suburbano & 19 & 19 \\
\hline & Rural & 9 & 95 \\
\hline Antecedentes & No AHF & 84 & 84 \\
\hline & Si AHF & 16 & 16 \\
\hline Quién es el familiar afectado & Padre & 2 & 2 \\
\hline & Madre & 2 & 2 \\
\hline & Hermanos & 3 & 3 \\
\hline & Otros & 9 & 9 \\
\hline
\end{tabular}

n: población, \%: porcentaje.

Fuente: Cuestionario general del estudio.

El rango de edad de los neonatos es de 4 a 28 días con una media de $14.7+7.9$; el peso al nacer fue de $1920 \mathrm{~g}$ a $4200 \mathrm{~g}$ con media de $3160.3+426.8 \mathrm{~g}$; el peso al mo- mento de la consulta está entre el rango de $2270 \mathrm{~g}$ y $5100 \mathrm{~g}$ con media de $3508.7 \mathrm{~g}$ $+586.3 \mathrm{~g}$. El resto de las características somatométricas están en la tabla 2. 


\section{Tabla 2}

Edad y características somatométricas de los neonatos, Celaya 2014.

\begin{tabular}{|lccccc|}
\hline Variable & $\mathrm{N}$ & $\mathrm{X}$ & $\mathrm{DS}$ & Min & Max \\
\hline Edad (días) & 100 & 14.7 & 7.9 & 4 & 28 \\
\hline Peso al Nacer $(\mathrm{g})$ & 100 & 3160.3 & 426.9 & 1320 & 4200 \\
\hline Peso a la Consulta $(\mathrm{g})$ & 100 & 3538.7 & 586.3 & 2270 & 5100 \\
\hline Estatura al Nacer $(\mathrm{cm})$ & 100 & 49.7 & 2.1 & 44 & 55 \\
\hline Estatura a la Consulta $(\mathrm{cm})$ & 100 & 51.9 & 2.6 & 47 & 60 \\
\hline
\end{tabular}

N: población, X: media, DS: desviación estándar, Min: mínimo, Max: Máximo.

Fuente: Cuestionario general del estudio y somatometría.

La validez para la prueba de transmisión del sonido en extensión / flexión con la sonda electroacústica se muestra en la tabla 3. Las tres mediciones realizadas se compararon con el diagnóstico por ultrasonido con técnica de Graf. Se obtuvieron sensibilidad, especificad y valores predicti- vos positivo y negativo muy elevados debido a que evalúan cada cadera por separado realizando el diagnóstico con mayor precisión. No hay diferencias significativas entre las mediciones para los diferentes parámetros de validez.

\section{Tabla 3}

\section{Validez de la prueba de extensión flexión con sonda electroacústica, Celaya, 2014}

\begin{tabular}{|c|c|c|c|c|c|c|c|}
\hline \multicolumn{2}{|c|}{$\begin{array}{l}\text { Transmisión del sonido } \\
\text { extensión flexión }\end{array}$} & $\begin{array}{l}\text { USG } \\
+\end{array}$ & $\begin{array}{c}\text { USG } \\
-\end{array}$ & $\begin{array}{l}\text { Sensibilidad } \\
\%(I C 95 \%)\end{array}$ & $\begin{array}{l}\text { Especificidad } \\
\%(I C 95 \%)\end{array}$ & $\begin{array}{c}\text { VPP } \\
(\text { IC95\%) }\end{array}$ & VPN (IC95\%) \\
\hline $1^{\circ}$ Medición & $\begin{array}{l}\text { TCSE/F + } \\
\text { TCSE/F - }\end{array}$ & $\begin{array}{l}24 \\
5\end{array}$ & $\begin{array}{c}1 \\
170\end{array}$ & $\begin{array}{c}82.76 \\
(67.29-98.23)\end{array}$ & $\begin{array}{c}99.42 \\
(97.98-100)\end{array}$ & $\begin{array}{c}96.00 \\
(86.32-100)\end{array}$ & $\begin{array}{c}97.14 \\
(94.39-99.90)\end{array}$ \\
\hline $2^{\circ}$ Medición & $\begin{array}{l}\text { TCSE/F + } \\
\mathrm{TCSE} / \mathrm{F} \text { - }\end{array}$ & $\begin{array}{l}24 \\
5\end{array}$ & $\begin{array}{c}0 \\
171\end{array}$ & $\begin{array}{c}82.76 \\
(67.29-98.23)\end{array}$ & $\begin{array}{c}100.0 \\
(99.71-100)\end{array}$ & $\begin{array}{c}100.0 \\
(97.92-100)\end{array}$ & $\begin{array}{c}97.16 \\
(94.42-99.90)\end{array}$ \\
\hline $3^{\circ}$ Medición & $\begin{array}{l}\text { TCSE/F + } \\
\text { TCSE/F3- }\end{array}$ & $\begin{array}{l}25 \\
4 \\
\end{array}$ & $\begin{array}{c}0 \\
171\end{array}$ & $\begin{array}{c}86.21 \\
(71.93-100) \\
\end{array}$ & $\begin{array}{c}100.0 \\
(99.71-100) \\
\end{array}$ & $\begin{array}{c}100.0 \\
(98.00-100)\end{array}$ & $\begin{array}{c}97.71 \\
(95.21-100) \\
\end{array}$ \\
\hline
\end{tabular}

USG: ultrasonido, TCSE/F: transmisión comparada del sonido en extensión/flexión, IC95\%: intervalo de confianza al 95\%, VPP: valor predictivo positivo, VPN: valor predictivo negativo.

Fuente: Mediciones con sonda electroacústica en extensión/flexión y ultrasonido de caderas.

La confiabilidad para la prueba de transmisión del sonido en extensión flexión con la sonda se presenta en la tabla 4. Se en- contró excelente confiabilidad intra-observador e inter-observador. 


\section{Tabla 4}

Confiabilidad inter e intra-observador para la sonda electroacústica, Celaya 2014.

\begin{tabular}{|c|c|c|c|c|c|}
\hline & & Segunda medición & TCSE/F & Tercera medición & TCSE/F \\
\hline & & + & - & + & - \\
\hline $\begin{array}{l}\text { Primera } \\
\text { medición } \\
\text { TCSE/F }\end{array}$ & $\begin{array}{l}+ \\
-\end{array}$ & $\begin{array}{c}24 \\
0\end{array}$ & $\begin{array}{c}1 \\
175\end{array}$ & $\begin{array}{c}24 \\
1\end{array}$ & $\begin{array}{c}1 \\
174\end{array}$ \\
\hline Kappa (IC & & \multicolumn{2}{|c|}{$0.98(0.93-1.0)$} & \multicolumn{2}{|c|}{$0.95(0.89-1.0)$} \\
\hline
\end{tabular}

TCSE/F: transmisión comparada del sonido en extensión/flexión, IC95\%: intervalo de confianza al 95\%.

Fuente: Mediciones con sonda electroacústica en extensión/flexión y ultrasonido de caderas

Con el ultrasonido de caderas con técnica de Graf se obtuvieron para el lado izquierdo 85 caderas sanas (Graf1), 13 caderas con inmadurez fisiológica (Graf 2) y 2 con subluxación (Graf 3). Para las caderas derechas, 86 fueron sanas (Graf 1) y 14 con inmadurez fisiológica (Graf 2).

\section{| | | | | | | | | | | | | | | | | | | | | | | | | | | | | | | | | | | | | | | | | | | | | | | | | | | | | | | | | | | | | | | | | | | | | | | | | | | | | | | | | | | | | | | | | | | | | | | | ||}

\section{Discusión}

La muestra no fue representativa de la población de los neonatos ya que se constituyó por invitación y de manera voluntaria, además, la frecuencia de antecedentes heredofamiliares $(16 \%)$ puede implicar un sesgo para la participación; lo anterior impide la generalización de los resultados, siendo esto una desventaja para el estudio. La validez de la TCSE/F con sonda electroacústica tiene porcentajes superiores al $80 \%$ para la sensibilidad, al $90 \%$ para especificidad, y valores predictivos superiores al 96\%; los resultados son simila- res al estándar de oro USG Graf utilizado para el cálculo de la validez. En el estudio de Kwong (2003) ${ }^{14}$, se usó un dispositivo para medir la discrepancia de la transmisión del sonido en la cadera, tomando esto como referencia la TCSE/F con la sonda electroacústica tiene menor sensibilidad (100\% vs $90 \%$ ) pero mayor especificidad ( $75 \%$ vs $100 \%$ ). En cuanto a la confiabilidad, el valor de Kappa mayor a 0.95, para las mediciones 1-2 (intra-observador) y 1-3 (inter-observador), indica que es una prueba altamente confiable no operador dependiente.

\section{Conclusiones}

El uso de la sonda electroacústica en TCSE/F complementa las pruebas clínicas habituales en el diagnóstico de DC, con la ventaja de ser una prueba que evalúa de manera objetiva la relación fisiológica de la articulación cabeza femoral-acetábulo, y permite detectar grados variables de displasia, desde la inmadurez fisiológica hasta la luxación. 


\section{Bibliografía}

1. William H. Hay Jr, Myron J. Levin, Judith M. Sondheimer, Robin R. Deterding. Current, Diagnosis and Treatment: Pediatrics. Colorado, USA: McGraw Hill. 2009.

2. Figueroa Ferrari RC, Padilla Raygoza N. Luxación congénita de cadera en el neonatos macrosómicos. Aspectos ultrasonográficos. Rev. Med. 1994; 32:277279.

3. Delgadillo JM, Macías Ha, Hernández R. Desarrollo displásico de cadera. Revista Mexicana de Pediatría, 2006:73 26-32.

4. American Academy Of Orthopaedic Surgeons, American Academy Of Pediatrics, The Pediatric Orthopaedic Society Of North America. Detection and nonoperative management of pediatric developmental dysplasia of the hip in infants up to six months of age. American Academy of Orthopeadic Surgeons. 2014; Junio 27, 2015, disponible en: http://www.aaos.org/research/ guidelines/DDHGuidelineFINAL.pdf

5. Dempsey M, Karmazyn B, Coley B, Dillman J, Dory C, Garber M, Hayes L, Milla S, Raske M, Rice H, Rigsby C, Strouse P, Westra S, Widmann R, Wootton-Gorges S. Developmental dysplasia of the hip. American College of Radiology. ACR appropriateness criteria. 2013; Julio 02, 2015, disponible en: http://www.guideline.gov/content.aspx?id $=4767$.

6. Shipman SA, Helfand M., Moyer V.A., Yawn B.P. Screening for developmental dysplasia of the hip: a systematic literature review for the US Preventive Services Task Force. Pediatrics. 2006; 117:557-576.

7. Storer SK, Skaggs DI. Developmental dysplasia of the hip. American Family Physician. 2006; 74:1310-1316.
8. García M, Guzmán JM, Dávalos ML, Escudero DA, Lavadores Al, Martínez SI, Rabago MR, Ríos R, Valenzuela $A$. Diagnóstico y tratamiento oportuno de la displasia del desarrollo de cadera. Centro Nacional de Excelencia Tecnológica en Salud. 2013; Julio 5, 2015, disponible en: http://www.cenetec.salud.gob.mx/ descargas/gpc/CatalogoMaestro/091_GPC_Displasiacaderaped/SSA_091_08_EyR.pdf.

9. Coran Arnold, Scott N. Aszick, Labarge Jean-Martín, Shamberger Robert, Caldamone Anthony. Pediatric surgery. Philadelphia, US.7a Edicion. Elsevier, Saunders.2012 pág. 1699-1710

10. Pillai A, Joseph J, Mcauley A, Bramley D. Diagnostic accuracy of static graf technique of ultrasound evaluation of infant hips for developmental dysplasia. Arch Orthopaedic Trauma Surgery. 2011; 131:53-58.

11. Finbogasson T. Dynamic ultrasonography in neonatal hip instability and acetabular dysplasia. Instituto Karolinska, Estocolmo, Suecia. 2008; Julio 02, 2015, disponible en:

https://openarchive.ki.se/xmlui/bitstream/handle/10616/38060/thesis.pdf?sequence $=1$.

12. Padilla $N$, Diaz $R$, Ruiz $M L$, Cordova $T$, Sosa $M, P e-$ rez $\mathrm{AH}$. Validity and reliability of a measuring device based on sound transmission for diagnosis of hip dysplasia in newborns. Scientific Research. 2014; Julio 15, 2015, disponible en: http://www.scirp.org/journal/ abb.

13. Ortega $X$. Displasia del desarrollo de la cadera. Rev. Med. Clin. Condes. 2013; 24:37-49.

14. Kwong KSC, Huang X, Cheng Jcy, Evans JH. New technique for early screening of developmental dysplasia of the hip: pilot study. Pediatr Orthop. 2003; 23: 347-351. 